\title{
CDISC Malaria Therapeutic Area User Guide Version 1.0
}

National Cancer Institute

\section{Source}

National Cancer Institute. CDISC Malaria Therapeutic Area User Guide Version 1.0. NCI

Thesaurus. Code C161447.

The 1.0 version of the CDISC Malaria therapeutic area user guide. 